\title{
Dynamic distractor environments reveal classic visual field anisotropies for judgments of temporal order
}

\author{
John Cass ${ }^{1}$ - Erik Van der Burg ${ }^{2,3}$
}

Published online: 5 December 2018

(C) The Psychonomic Society, Inc. 2018

\begin{abstract}
Numerous studies have shown that visual performance critically depends on the stimulus' projected retinal location. For example, performance tends to be better along the horizontal relative to the vertical meridian (lateral anisotropy). Another case is the so-called upper-lower anisotropy, whereby performance is better in the upper relative to the lower hemifield. This study investigates whether temporal order judgments (TOJs) are subject to these visual field constraints. In Experiments 1 and 2, subjects reported the temporal order of two disks located along the horizontal or vertical meridians. Each target disk was surrounded by 10 black and white distractor disks, whose polarity remained unchanged (static condition) or reversed throughout the trial (dynamic condition). Results indicate that the mere presence of dynamic distractors elevated thresholds by more than a factor of four and that this elevation was particularly pronounced along the vertical meridian, evidencing the lateral anisotropy. In Experiment 3, thresholds were compared in upper, lower, left, and right visual hemifields. Results show that the threshold elevation caused by dynamic distractors was greatest in the upper visual field, demonstrating an upper-lower anisotropy. Critically, these anisotropies were evident exclusively in dynamic distractor conditions suggesting that distinct processes govern TOJ performance under these different contextual conditions. We propose that whereas standard TOJs are processed by fast low-order motion mechanisms, the presence of dynamic distractors mask these low-order motion signals, forcing observers to rely more heavily on more sluggish higher order motion processes.
\end{abstract}

Keywords Temporal order · Apparent motion · Attention

\section{Introduction}

Our ability to extract information from the visual environment depends upon which regions of retina are stimulated. A potent example of this is the loss of spatial acuity that occurs with increasing visual eccentricity (Golla, Ignashchenkova, Haarmeier, \& Thier, 2004; Levi \& Waugh, 1994; Seiple, Holopigian, Szlyk, \& Wu, 2004; Virsu \& Rovamo, 1979; Yeshurun \& Carrasco, 1999). In an attempt to equate stimulus visibility, psychophysical researchers routinely place stimuli at isoeccentric locations. Not all isoeccentric locations afford

John Cass

j.cass@westernsydney.edu.au

1 School of Social Sciences \& Psychology, Western Sydney University - Bankstown Campus, Milperra, Australia

2 Department of Experimental \& Applied Psychology, Vrije Universiteit, Amsterdam, Netherlands

3 School of Psychology, University of Sydney, Sydney, Australia equivalent performance, however. Contrast sensitivity and spatial acuity, for instance, tend to be enhanced in the lower visual field. This upper-lower anisotropy (also known as the vertical anisotropy) (Carrasco, Talgar, \& Cameron, 2001; Corbett \& Carrasco, 2011) is at least partially explained by a greater abundance of retinal ganglia in superior retina (Curcio \& Allen, 1990; Perry \& Cowey, 1985). Lower visual field performance advantages also exist for tasks which presumably invoke higher level processing, including conjunction search (Carrasco, Giordano, \& McElree, 2004; Chaikin, Corbin, \& Volkmann, 1962; Kristjansson \& Sigurdardottir, 2008); object individuation (reduced crowding) and multiple object tracking ( $\mathrm{He}$, Cavanagh, \& Intriligator, 1996; Intriligator \& Cavanagh, 2001).

Another well-documented anisotropy, known as the lateral anisotropy (also known as the horizontal-vertical anisotropy), refers to the finding that performance along the horizontal meridian (east and west of fixation) generally tends to be better than along the vertical meridian (north and south; Cameron, Tai, \& Carrasco, 2002; Carrasco, Evert, Chang, \& Katz, 1995; Carrasco \& Frieder, 1997; Corbett \& Carrasco, 2011; Rijsdijk, Kroon, \& van der Wildt, 1980; Virsu \& Rovamo, 
1979). Like the upper-lower anisotropy, evidence for the lateral anisotropy has been found across a range of low-level and highlevel visual tasks, including contrast detection (Cameron et al., 2002), spatial acuity (Talgar \& Carrasco, 2002), and visual search (Abrams, Nizam, \& Carrasco, 2012).

In addition to these performance advantages (and disadvantages) conferred by certain regions of the visual field, the rate at which visual information is processed has been found to obey analogous visual field constraints, with contrast and spatial judgments being processed more rapidly along horizontal than along vertical meridian locations (Carrasco et al., 2004; Corbett $\&$ Carrasco, 2011). Carrasco et al. (2004) have dubbed such anisotropies in processing rate 'temporal performance fields'.

But what about judgments pertaining not to the processing of static luminance and spatial form but to temporal aspects of the stimulus? A fundamental factor limiting our sensitivity to the timing of visual events is critical flicker fusion (CFF): the maximum rate of flicker at which observers are able to detect the presence of temporal modulation (de Lange, 1952, 1958). Unlike the performance anisotropies described above, sensitivity to high frequencies moderately improves with eccentricity (Raninen \& Rovamo, 1997). Evidence that CFF exhibits an upper-lower anisotropy is mixed, although most studies report higher temporal resolution in the lower relative to the upper visual field (Tyler, 1987; but see Rovamo \& Raninen, 1984; Yasuma, Miyakawa, \& Yamazaki, 1986).

Whereas CFF judgments involve visual analysis of a single object, other timing judgments require temporal analysis of multiple objects. One such task, known as temporal phase discrimination, involves two objects each engaged in periodic temporal modulation at a common frequency, but variable phase relationship (Forte, Hogben, \& Ross, 1999; RogersRamachandran \& Ramachandran, 1998). Phase acuity thresholds are then extracted by plotting phase discrimination performance as a function of stimulus modulation rate. Temporal acuity for this task has been found to degrade asymptotically as a function of the spatial distance between the pair of modulating objects (Aghdaee \& Cavanagh, 2007). The authors interpret this Performance $\times$ Distance Dependency interaction as implying the existence of two perceptual systems: (i) a short-range first-order motion-sensitive system capable of high temporal resolution $(\sim 20-40 \mathrm{~Hz})$, coupled with (ii) a long-range, higher order, motion-tracking system operating with an average temporal resolution of $8.9 \mathrm{~Hz}$ at separations $\geq 4$ degrees of visual angle. Interestingly, their results show that whilst lower visual fields afforded higher acuity at the smallest interelement separations, no such anisotropy was found at larger separations.

According to Aghdaee and Cavanagh's (2007) dual processing scheme, their results imply a dissociation whereby the more locally constrained first-order motion system appears to express the expected upper-lower anisotropy (higher temporal resolution in the lower visual field-although no mention was made of this feature of their data in their original paper), whilst the more sluggish and longer range motiontracking system appears to be roughly isotropic.

A more recent phase discrimination study calls this interpretation into question, however, showing significant degradation in phase discrimination performance between $10^{\circ}$ and $100^{\circ}$ of visual separation (Maruya, Holcombe, \& Nishida, 2013), far more extensive than the maximum $16^{\circ}$ separation tested by Aghdaee and Cavanagh (2007). Maruya and colleagues' result suggests that manipulating interelement separation is unlikely to completely 'silence' the contribution of early motion filters to temporal phase judgments. Consequently, the idea that phase judgments regarding pairs of stimuli that are spatially remote reflect exclusively the processing of a high-order tracking system is questionable.

Another task commonly used to estimate the visual system's temporal resolution is the temporal order judgment (TOJ). In this paradigm, a single temporal offset is introduced between two spatially distinct events (typically a luminance increment or decrement), and it is the observers' task to correctly sequence them. By plotting these responses as a function of the magnitude and sign of this temporal displacement, one can determine temporal precision using the slope of the fitted psychometric function.

Like phase judgments, judgments of temporal order could conceivably be mediated by a combination of a fast, first-order motion system (albeit long range) and a more sluggish, higher order tracking system (Holcombe, 2009). Recently we introduced a new paradigm to effectively reduce the contribution of this ostensibly faster low-order motion system to TOJs (Cass \& Van der Burg, 2014). This technique, known as remote temporal camouflage (RTC), involves a standard visual TOJ task performed in the context of dynamic (abruptly modulating) distractor events. We have shown previously that the mere presence of abrupt distractor events - even at locations that are spatially remote from their neighbouring targets - can profoundly interfere with TOJ precision, with thresholds increasing from approximately $25 \mathrm{~ms}$ in static (nonmodulating) distractor contexts, to more than $80 \mathrm{~ms}$ in the presence of dynamic (modulating) distractors (Cass \& Van der Burg, 2014; Talbot, Van der Burg, \& Cass, 2017).

This effect of dynamic distractors on TOJ thresholds is consistent with the dual processing scheme for motion. The 'faster' of these systems distinguishes target temporal order by comparing displacements in overall first-order motion energy. Whilst this system is likely to be efficient in static distractor environments, where transient signal to noise is low, it is likely to fail if sufficient levels of transient noise are introduced. This, we propose, is what occurs in dynamic distractor environments, whereby the differential response of directionselective and target-relevant motion filters (signal) is subsumed - at least partially — by the net motion elicited by irrelevant distractor-driven responses (noise). It is under these 
transient distractor masking environments that we assume that the visual system relies more heavily on high-order attentional-tracking mechanisms to perform TOJs.

In this study, we apply the RTC paradigm to the question of whether there might exist visual anisotropies for judgments of temporal order that may have been indiscernible using previous psychophysical methods. Few reports exist on whether there are visual anisotropies for judgments of temporal order. Those that have investigated this report no evidence for any lateral anisotropy (Lim \& Sinnett, 2012; Westheimer, 1983). To our knowledge, TOJ performance has not been evaluated in upper versus lower visual fields.

Experiments 1 and 2 look for evidence of the lateral anisotropy for TOJs measured in both static and dynamic distractor environments. Experiment 3 uses this approach to investigate the upper-lower anisotropy. Based on our previous work, we expect dynamic distractor environments will produce strong TOJ threshold elevation relative to static environments. If the mechanism(s) supporting the high-order tracking system are common to those observed in other high-order task domains (e.g. visual search, individuation, tracking, crowding), we predict that TOJ performance in dynamic distractor conditions will match qualitatively the strong anisotropies observed previously in these various tasks. If, however, they operate independently of one another, then we see no reason to expect the same set of anisotropies (lateral and horizontal-vertical)—or in fact, any anisotropy at all.

\section{Experiment 1}

\section{Method}

\section{Observers}

Nine human observers (six females, three males) with ages ranging from 22 to 42 years participated in all experiments after giving informed written consent. All were naïve to the purposes of the experiment and were paid \$AUD 25 per hour for their participation. All had normal or corrected-to-normal vision. Experiments were approved by the University of Western Sydney's Human Research Ethics committee (approval number H8862) and were conducted in accordance with the Code of Ethics of the World Medical Association (Declaration of Helsinki) for experiments involving humans.

\section{Apparatus and stimuli}

Stimuli were created using E-Prime running on a desktop PC. Stimuli were presented on an LCD monitor (Viewsonic VX2265wm; $1024 \times 768$ pixels, $85 \mathrm{~Hz}$ ). Viewing distance was $57 \mathrm{~cm}$. In all experiments the screen's background was grey and its luminance was held constant at $32 \mathrm{~cd} / \mathrm{m}^{2}$.

\section{Procedure}

Each trial began with a single white circular fixation point (diameter $=0.2^{\circ}, 62 \mathrm{~cd} / \mathrm{m}^{2}$ ) presented at the centre of the screen for 1 second. Four black target disks (diameter $=1.5^{\circ}$ of visual angle, $2 \mathrm{~cd} / \mathrm{m}^{2}$ ) appeared: one $8^{\circ}$ to the left, to the right, above, and below fixation. Each target disk was surrounded by a set of 10 distractor disks $\left(\right.$ diameter $=1.5^{\circ}$ ), with each set of distractors equidistantly located on an imaginary circle (radius $=3^{\circ}$ ) centred on the target disk. Each distractor disk was randomly assigned to be either black (2 $\left.\mathrm{cd} / \mathrm{m}^{2}\right)$ or white $\left(62 \mathrm{~cd} / \mathrm{m}^{2}\right)$ at the beginning of each trial. Two general distractor conditions were used across the experiments: static and dynamic distractor contexts. In the dynamic context, distractor ensembles surrounding horizontally arranged and vertically arranged targets, each changed a total of 19 times (see Fig. 1 for a time-course schematic of horizontally distributed target/distractor ensembles). The initial set of distractor changes involved between two and five distractor disk(s) undergoing abrupt changes in luminance polarity, with each change separated in time by a randomly determined interval $(47 \mathrm{~ms}, 94 \mathrm{~ms}$, or $153 \mathrm{~ms})$. These distractor changes continued until a randomly determined number of events had occurred (14-17). Subsequently, after $47 \mathrm{~ms}$, the luminance of one of the four target disks changed abruptly from black to white, then, after a randomly determined SOA, an equivalent luminance change occurred in the target at the opposite side of fixation. That is to say, a luminance change in the left target would be followed by a luminance change in the right target, or vice versa. By contrast, a luminance change in the bottom target would be followed by a change in the top target, or vice versa. On trials where target-related luminance changes occurred in a horizontally displaced target, no change would occur in a vertically displaced target, and vice versa. The SOAs used in all conditions were: $-294 \mathrm{~ms},-247 \mathrm{~ms},-94$ $\mathrm{ms},-59 \mathrm{~ms},-35 \mathrm{~ms},-12 \mathrm{~ms}, 12 \mathrm{~ms}, 35 \mathrm{~ms}, 59 \mathrm{~ms}, 94 \mathrm{~ms}, 247$ $\mathrm{ms}$, and $294 \mathrm{~ms}$. Negative SOAs indicate that the left or the top target changed first, whereas positive SOAs indicate that the right or bottom target changed first. In the case of the static distractor condition, $153 \mathrm{~ms}$ after the onset of the first target event, the remaining distractor changes occurred. In the case of the dynamic distractor condition the remaining distractor changes occurred $306 \mathrm{~ms}$ after the onset of the first target. Similar to the previous distractor changes, a randomly determined number (two to five) of distractor disks were assigned to undergo an abrupt change in luminance polarity (from black to white or reversed) to produce a total of 19 distractor changes along both horizontally and vertically displaced distractors. In the static contextual condition, the luminance of the distractors remained constant before disappearing at the conclusion of the trial. Aside from the distractor changes, the timing of the static distractor condition was identical to the dynamic condition. Following a key-press response, the 

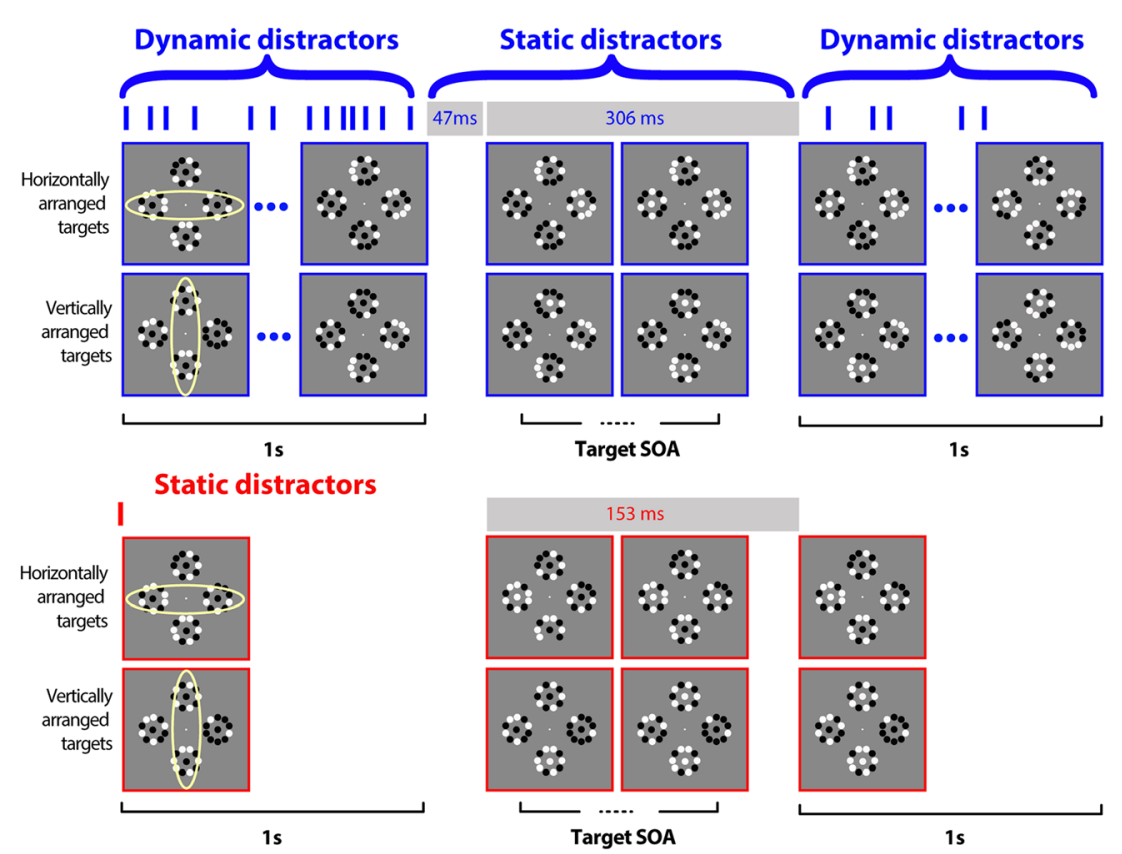

Fig. 1 Example trial sequence representing each of the two distractor conditions and target meridians used in Experiment 1: dynamic context (blue); static context (red). Note that in the dynamic context distractor elements remained unchanged for $47 \mathrm{~ms}$ prior to the first target event. The interval between subsequent dynamic distractor events (prior to or following the target events) was either 47,94 , or $153 \mathrm{~ms}$, chosen randomly following each distractor event. The distractor events during the course of

a trial are indicated by vertical bars above the example displays. The yellow ellipses in the left panels, presented here for illustrative purposes, signify whether the trial involved target events presented along the horizontal or the vertical meridian. The two target events were always presented on either the horizontal or the vertical meridian. (Colour figure online)

display was set to mean grey, and the next trial was initiated after a $300 \mathrm{~ms}$ intertrial period. Each SOA was presented 16 times in each contextual condition (768 trials in total per subject). On trials containing vertically arranged target changes, horizontally arranged targets remained black throughout the trial. Conversely, vertically arranged targets remained black throughout horizontal target trials. The subjects' task was to identify whether the left, right, bottom, or top target event occurred first, by pressing the leftward, rightward, downward or upward arrow keys, respectively. The order of conditions (target SOA, distractor context, and meridian) varied randomly from trial to trial. No cue was provided to indicate which set of targets (horizontal or vertical meridian) would undergo luminance transition on a given trial. All observers completed a practice session consisting of 24 trials to familiarise them with the task.

\section{Analysis}

Just noticeable differences (JNDs) were obtained in each condition by fitting a cumulative Gaussian separately to each observer's data (proportion of 'right first' or 'bottom first' responses as a function of target SOA) using a LevenburgMarquardt algorithm maximum likelihood fitting procedure and multiplying the fitted standard deviation of this fit by 0.6745 (Cass \& Van der Burg, 2014; Rodríguez-Sánchez, Fallah, \& Leonardis, 2016). The cumulative offset parameter

(equivalent to the point of subjective simultaneity; PSS) was free to vary.

\section{Results}

A chi-square analysis was conducted on each individual participant's data (averaged across SOA) to estimate the goodness of fit for each experimental condition. Respectively, the average $\chi^{2}$ and $p$ values (7 degrees of freedom) obtained in each condition were static vertical, $\chi^{2}=16.4, p=.04$; static horizontal, $\chi^{2}=14.6, p=.07$; dynamic vertical, $\chi^{2}=19.0, p=.16$; dynamic horizontal, $\chi^{2}=19.6, p=.13$. Given that a proportion of these psychometric fits failed to reach critical significance, we ran a series of confirmatory analyses comparing the proportion of 'correct' responses for negative versus positive SOAs ms (omitting the two smallest absolute SOAs) in the various conditions. Note that for the most part, these accuracy effects (reported below) qualitatively match the JND analyses below.

Just noticeable difference (JND) The results of Experiment 1 are shown in Fig. 2. A repeated-measures ANOVA on JNDS reveals a significant main effect of distractor context, $F(1,8)=$ $19.625, p=.002, \eta^{2}=.710$, with JNDs measured in dynamic distractor contexts more than 8 times greater on average (216 $\mathrm{ms})$ than those in static distractor environments $(25 \mathrm{~ms})$. A significant main effect of meridian was also observed, $F(1$, $8)=8.582, p=.019, \eta^{2}=.518$, with targets positioned on the 


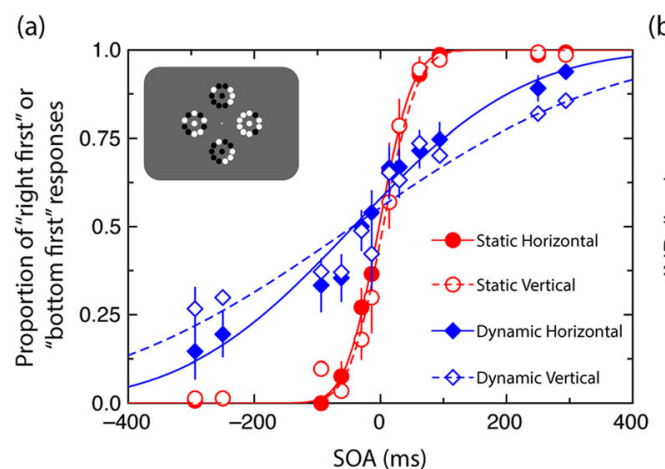

(b)

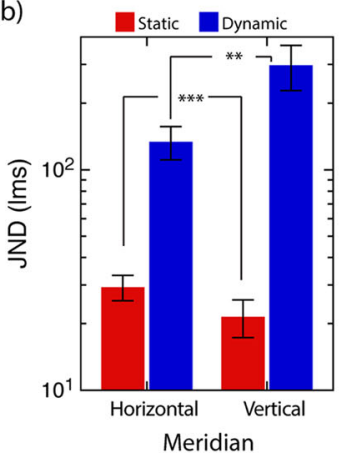

(c)

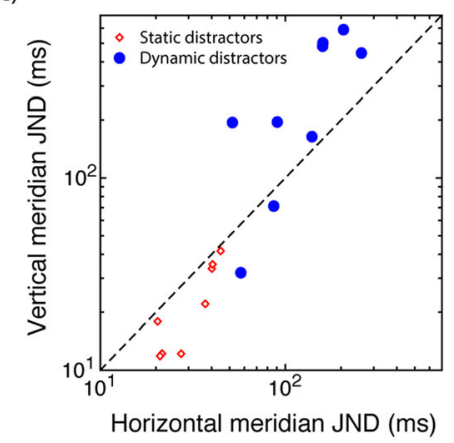

Fig. 2 Results of Experiment 1. a Proportion of 'right target first' or 'bottom target first' responses as a function of target stimulus onset asynchrony (SOA) averaged across subjects for each of the two distractor and meridian conditions: static distractors (red circles); dynamic distractors (blue diamonds); horizontal meridian (filled symbols); vertical meridian (open symbols). Curves are best-fitting cumulative Gaussians measured for each contextual condition. Inset image shows the stimulus configuration. b Average JNDs derived from individual subjects' fits in each contextual distractor and meridian condition. Error bars represent between-subject mean standard errors. c Comparison of individual observer JNDs measured along horizontal and vertical meridians under dynamic (blue circles) and static (red diamonds) distractor conditions. Note that the dynamic JNDs located above the diagonal indicate a lateral anisotropy asymmetry for these observers. (Colour figure online) vertical meridian yielding average thresholds more than twice those measured along the horizontal meridian (298 ms vs 134 $\mathrm{ms})$. The ANOVA yielded a significant interaction between distractor context and meridian, $F(1,8)=10.929, p=.011$, $\eta^{2}=.577$. This interaction was further examined by two separate one-tailed $t$ tests (Bonferroni adjusted) for each type of distractor. In the dynamic context, the $t$ test yielded significant poorer performance (JNDs $163 \mathrm{~ms}$ higher, on average) along the vertical meridian than along the horizontal meridian, $t(8)=$ $-3.117, p=.014$. A significant but opposite meridian effect was observed in the static distractor context, with the vertical meridian yielding thresholds $8 \mathrm{~ms}$ lower on average than along the horizontal meridian, $t(8)=-4.989, p=.001$. Confirmatory repeated-measures $t$ tests run on accuracy data yielded qualitatively equivalent meridian effects for the dynamic distractor condition, $t(8)=4.850, p<.001$; but not the static distractor condition, $t(8)=1.367, p=.208$. For illustrative purposes, a comparison of JNDs derived along horizontal and vertical meridians for individual observers is depicted in Fig. 2c. Note that for the dynamic distractor conditions a visible anisotropy was evident for at least six of the nine observers.

Point of subjective simultaneity (PSS) A repeated-measures ANOVA on PSS estimates found no evidence for any significant main effects of distractor context, $F(1,8)=3.728, p=$ $.090, \eta^{2}=.318$, or meridian, $F(1,8)=0.178, p=.685, \eta^{2}=$ .022 . No evidence was found for the two-way interaction, $F(1$, 8) $=0.426, p=.532, \eta^{2}=.051$.

A series of four one-sample $t$ tests was conducted to determine whether any of the four conditions produced PSS estimates differing significantly from zero. Table 1 shows that no experimental condition produced PSS estimates deviating from zero.

\section{Discussion}

Consistent with our previous studies (Cass \& Van der Burg, 2014; Talbot et al., 2017), Experiment 1 shows that the mere presence of abruptly modulating distractors produces profound threshold elevation ( $\sim 850 \%$ on average). Overall, thresholds were lower when targets were positioned along the horizontal meridian (east and west of fixation) relative to when they were arranged vertically (north and south). This lateral anisotropy was evident exclusively in dynamic distractor environments. By contrast-and somewhat unexpectedly - an opposite anisotropy was observed in the static condition, with mean thresholds $8 \mathrm{~ms}$ lower along the vertical relative to the horizontal meridian.

This dissociation between the effects of distractor dynamics and visual meridian supports the idea that TOJ performance is mediated by at least two partially independent motion systems (Holcombe, 2009): (i) a 'fast' system capable of differentiating the direction of low-order motion signals, dominating in static environments; and (ii) a more sluggish, possibly higher level positional tracking system, dominating in dynamic distractor environments. One must be cautious in this interpretation, however. Human observers produce strong biases in saccadic scanning

Table 1 Mean PSS estimates and results of one-sample $t$ tests showing that all conditions in Experiment 1 yielded PSSs statistically indistinguishable from zero milliseconds

\begin{tabular}{llll}
\hline Condition & Mean PSS (ms) & $t$ & $p$ \\
\hline Static horizontal & -4.6 & -1.360 & .211 \\
Static vertical & 2.3 & .314 & .761 \\
Dynamic horizontal & -41.7 & -1.668 & .134 \\
Dynamic vertical & -85.7 & -1.200 & .265 \\
\hline
\end{tabular}


behaviour consistent with the horizontal layout of natural scenes (Foulsham, Kingstone, \& Underwood, 2008). The superior performance obtained along the horizontal meridian (in the dynamic condition) may therefore, conceivably result from a cognitive bias to preferentially attend to objects located along the horizontal meridian relative those arranged on the vertical meridian.

\section{Experiment 2}

To address this possibility, we conducted a second experiment using a blocked design in which targets were always located along the same visual meridian within a given block of trials. It is anticipated that this will reduce to almost zero any spatial uncertainty subjects might have about the location of the target events on any given trial. Hence, they will be less inclined to simply ignore the vertical meridian as they may do when presented with the four potential target locations used in Experiment 1.

We note that the average magnitude of threshold elevation in Experiment 1 far exceeds that of our original study (Cass \& Van der Burg, 2014). In addition to increased uncertainty about the likely location of targets on a given trial, the total number of distractors presented within any given trial in Experiment 1 exceeds our previous study by a factor two. In Experiment 2 we remove this uncertainty by halving the number of targets and distractors so that on a given trial (and within a given experimental block) a single pair of target/distractor ensembles appears along either the horizontal or the vertical meridian (see Fig. 3 for example stimulus configurations).

\section{Method}

\section{Observers}

Nine human observers (three females, six males) with ages ranging from 21 to 42 years participated in this experiment. Two were authors. The remaining seven were naïve to the purposes of the experiment and did not participate in the other experiments. Each provided informed written consent and was paid \$AUD 25 per hour for their participation. One female observer also participated in Experiment 1. All had normal or corrected-to-normal vision. Experiments were approved by the University of Western Sydney's Human Research Ethics committee (H8862) and were conducted in accordance with the Code of Ethics of the World Medical Association (Declaration of Helsinki) for experiments involving humans.

\section{Apparatus, stimuli, and procedure}

Experiment 2 was identical to Experiment 1, with the following exceptions. The SOAs employed in this experiment were -94 $\mathrm{ms},-59 \mathrm{~ms},-35 \mathrm{~ms},-12 \mathrm{~ms}, 12 \mathrm{~ms}, 35 \mathrm{~ms}, 59 \mathrm{~ms}$, and $94 \mathrm{~ms}$. On each trial, only a single pair of target/distractor ensembles was ever presented. Moreover, within an experimental block of trials, targets were always presented along the same meridian (either horizontal or vertical). (For stimulus configurations, refer to Fig. 3). Each meridian block consisted of 256 trials per subject (16 trials per SOA per condition), and each subject performed two blocks of trials (one for each meridian). Order of

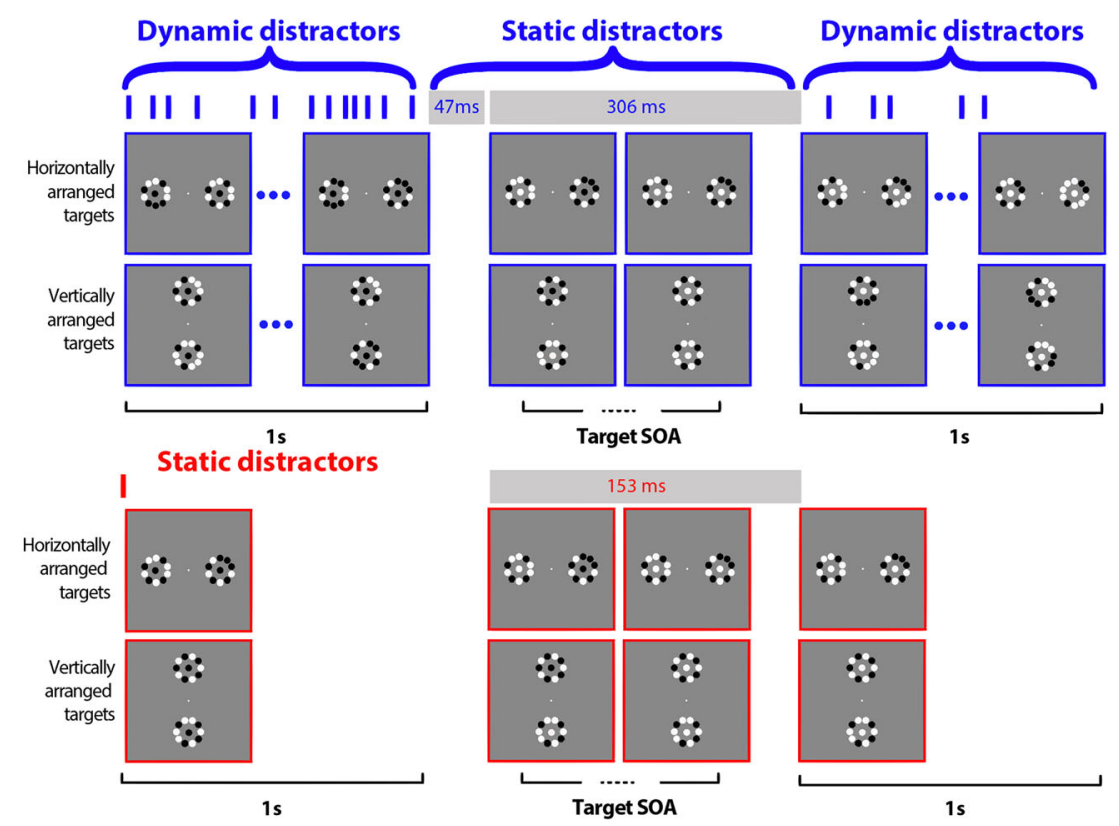

Fig. 3 Example trial sequence representing each of the two contextual conditions presented along each visual meridian used in Experiment 2: Dynamic context (blue); static context (red). Both the visual meridian and

distractor condition was randomly selected across trials. Blue and red vertical bars above the stimulus configuration panels signify distractor events. (Colour figure online) 
meridian blocks was counterbalanced across observers. Within an experimental block, distractor context (static vs dynamic distractors) and target SOA were randomised from trial to trial.

\section{Results}

A chi-square analysis was conducted on each individual participant's data (averaged across SOA) to estimate the goodness of fit for each experimental condition. Respectively, the average $\chi^{2}$ and $p$ values (7 degrees of freedom; measured SOAs -1$)$ obtained in each condition were static vertical, $\chi^{2}$ $=15.1, p=.38$; static horizontal, $\chi^{2}=18.4, p=.02$; dynamic vertical, $\chi^{2}=18.0, p=.12$; dynamic horizontal, $\chi^{2}=14.6, p=$ .07. Like Experiment 1, a proportion of these psychometric fits failed to reach critical significance. Consequently, we ran a series of confirmatory analyses comparing the proportion of 'correct' responses for negative versus positive SOAs (omitting the two smallest absolute SOAs) in the various conditions. Note that these accuracy effects (reported below) all qualitatively match the JND analyses below.

Just noticeable difference (JND) The results of Experiment 2 are shown in Fig. 4. A repeated-measures ANOVA shows a significant main effect of distractor context, $F(1,8)=16.303, p$ $=.004, \eta^{2}=.671$, indicating that dynamic distractor environments induced significantly higher TOJ thresholds relative to static environments. A significant main effect of meridian was also observed in this experiment, $F(1,8)=30.993, p=.001, \eta^{2}$ $=.795$, with TOJ precision being significantly worse overall along vertical compared to horizontal meridian locations. A significant interaction between distractor context and meridian was also observed, $F(1,8)=27.290, p=.001, \eta^{2}=.773$. This interaction was further examined by two separate two-tailed $t$ tests for each distractor context. In the dynamic context, the $t$ test yielded significantly poorer performance along the vertical relative to the horizontal meridian, $t(8)=5.650, p<.001$. Unlike, Experiment 1 no meridian effect was observed in static distractor contexts, $t(8)=1.719, p=.124$. Confirmatory repeated-measures $t$ tests run on accuracy data yielded qualitatively equivalent effects: dynamic horizontal versus vertical, $t(8)=2.127, p=.033$; static horizontal versus vertical, $t(8)=$ $0.224, p=.586$. For illustrative purposes, a comparison of JNDs derived along horizontal and vertical meridians for individual observers is depicted in Fig. 4c.

Using meridian and context as within-subjects factors, a between-samples ANOVA comparing JNDs across Experiments 1 and 2 indicates that overall, JNDs were significantly lower in the latter experiment, $F(1,34)=16.118, p<$ $.001, \eta^{2}=.578$. We also observe a significant interaction between distractor context and experiment, $F(1,34)=11.992, p$ $=.001, \eta^{2}=.158$. Two separate independent-samples $t$ tests (two-tailed) showed that Experiment 2 yielded lower thresholds than did Experiment 1 in both static and dynamic distractor environments: $t \mathrm{~s}(34)=-2.858$ and $-3.405, p \mathrm{~s}=$ .007 and .002 , respectively. No significant interaction was observed between experiment and meridian $F(1,34)=$ 2.484, $p=.120, \eta^{2}=.037$, neither was there a significant three-way interaction between experiment, distractor context, and meridian, $F(1,34)=3.439, p=.068, \eta^{2}=.051$.

Point of subjective simultaneity (PSS) A repeated-measures ANOVA on PSSs derived from Experiment 2 failed to find a significant main effect of distractor context, $F(1,8)=2.225, p$ $=.174, \eta^{2}=.218$. A significant meridian effect was observed, $F(1,8)=13.242, p=.007, \eta^{2}=.623$, showing that when collapsed across both levels of distractor context, targets located along the vertical meridian produced larger (negative) shifts in PSS (average $=-26.5 \mathrm{~ms}$ ) than when located along (a)

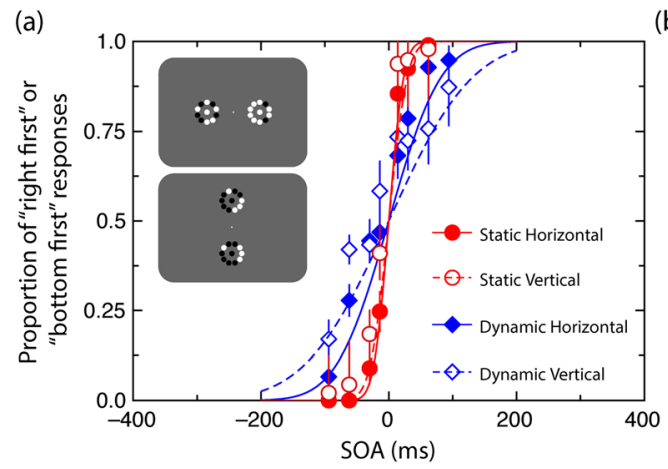

(b)

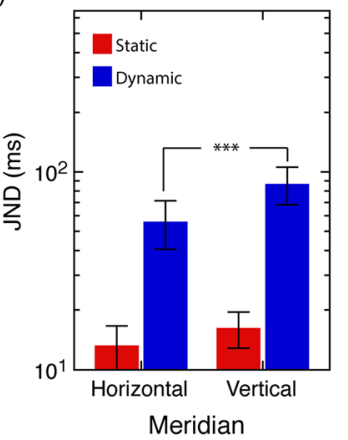

(c)

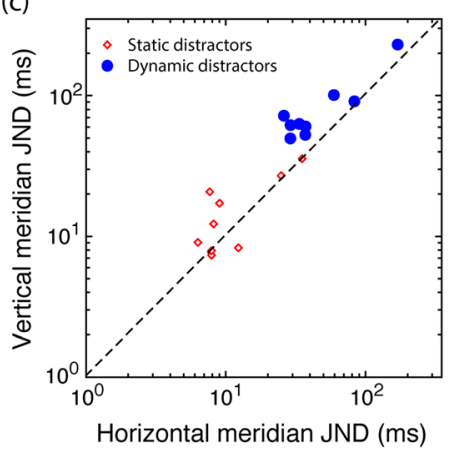

Fig. 4 Results of Experiment 2. a Proportion of 'right target first' or 'bottom target first' responses as a function of target stimulus onset asynchrony (SOA) averaged across subjects for each of the two distractor and meridian conditions: Static distractors (red circles); dynamic distractors (blue diamonds); horizontal meridian (filled symbols); vertical meridian (open symbols). Curves are best-fitting cumulative Gaussians measured for each contextual condition. Inset image shows the stimulus

configurations. b Average JNDs derived from individual subjects' fits in each contextual distractor and meridian condition. Error bars represent between-subject mean standard error. c Comparison of individual observer JNDs measured along horizontal and vertical meridians under dynamic (blue circles) and static (red diamonds) distractor conditions. Note that the dynamic JNDs located above the diagonal indicate a lateral anisotropy asymmetry for these observers. (Colour figure online) 
the horizontal meridian (average $=-16.07 \mathrm{~ms}$ ). A significant interaction between distractor context and meridian was not observed, $F(1,8)=0.404, p=.543, \eta^{2}=.048$ (Table 2)

\section{Discussion}

The results of Experiment 2 echo those of Experiment 1 in most respects. Again, the mere presence of dynamic distractor events caused profound TOJ threshold elevation. Importantly, this elevation was more pronounced for target/distractor ensembles distributed along the vertical (north-south) meridian relative to the horizontal (east-west). In this experiment, however, no anisotropy was observed in static distractor conditions.

Although the spatial distribution of threshold elevation was qualitatively similar in the two experiments, thresholds were not equivalent on average, with lower and less variable dynamic distractor-related thresholds in conditions employing fewer target and distractor elements (Experiment 2). The greater threshold elevation in Experiment 1 we believe may be due to greater spatial uncertainty, and possibly attentional undersampling, in the case of Experiment 1 (see Olivers, Awh, \& Van der Burg, 2016; Van der Burg, Awh, \& Olivers, 2013, for estimating the capacity to detect events in a dynamic context). Moreover, the more numerous distractors employed in Experiment 1 may have also contributed to the overall decrease in JNDs.

That our observed lateral anisotropy survived Experiment 2's blocked design (in dynamic distractor conditions) eliminates the possibility that it is due neither to uncertainty about the likely location of target events nor the tendency to ignore vertically arranged visual stimuli in favour of horizontal arrangements. Given the qualitative similarity between the lateral anisotropy observed in our dynamic distractor conditions and those reported in numerous nontemporal tasks, it seems reasonable to suggest that our RTC paradigm may yield evidence of the other classic, so-called upper-lower, performance field anisotropy.

\section{Experiment 3}

The TOJ task in this experiment is similar to that used in Experiments 1 and 2, with subjects reporting the temporal

Table 2 Mean PSS estimates and results of one-sample $t$ tests for Experiment 2, showing statistical deviation of PSS estimates from zero milliseconds. Double asterisks signify statistically significant $p$ values less than .025

\begin{tabular}{lllll}
\hline Distractor context & Visual meridian & Mean PSS (ms) & $t$ & \multicolumn{2}{l}{$p$} \\
\hline Static & Horizontal & -12.2 & -0.634 & .543 \\
& Vertical & -19.3 & -3.005 & $.017^{* *}$ \\
\multirow{2}{*}{ Dynamic } & Horizontal & -81.5 & -1.306 & .134 \\
& Vertical & -91.5 & -1.959 & .265 \\
\hline
\end{tabular}

sequence of horizontally or vertically arranged stimuli. Unlike Experiment 1, however, in which the virtual motion trajectory associated with each sequence pair passes through fixation, the target trajectories employed in Experiment 3 are either horizontal (above or below fixation) or vertical (left or right of fixation). This implies the existence of just two possible trajectory locations in Experiment 1 (up-down vs left-right through fixation), but four in Experiment 3 (horizontal above and below fixation vs vertical left and right of fixation). In addition to the increase in spatial uncertainty associated with the use of four possible target locations (as in Experiment 1), the introduction of four possible trajectory locations in Experiment 3 could conceivably introduce additional uncertainty. To mitigate this latter possibility and ensure an equivalent degree of spatial uncertainty to that invoked by Experiment 1 , for Experiment 3 we chose to present trials in blocks consisting of (i) those requiring horizontal analysis of temporal order (upper vs lower visual fields; see Fig. 6a), and (ii) those requiring vertical analysis (left vs right visual fields; see Fig. 6b).

We predict that if the mechanisms which support perception of high-level motion tracking are common to those responsible for performance in other attentionally demanding, nontemporal tasks (e.g. conjunction search, object individuation; He et al., 1996; Intriligator \& Cavanagh, 2001), then TOJ threshold elevation will be greater for horizontal TOJs positioned in the upper visual field relative to those in the lower visual field.

In addition to the upper-lower field anisotropy this experiment permits us to examine whether the horizontal vertical anisotropy observed in Experiments 1 and 2 is specifically linked to temporal acuity associated with the meridians themselves or whether it is the orientation of the virtual trajectories that determines performance. If stimulus orientation alone drives the lateral anisotropy observed in Experiments 1 and 2, then we expect vertical judgments (Fig. 6b) to produce higher thresholds than horizontal judgments (Fig. 6a). Alternatively, if the lateral anisotropy is specific to meridians themselves, then we have no reason expect vertical TOJs to yield significantly higher thresholds than horizontal TOJs.

\section{Method}

\section{Observers}

Fourteen human observers (10 females, four males) with ages ranging from 22 to 42 years participated in this experiment. Two were authors. The remaining 12 were naïve to the purposes of the experiment and did not participate in the other experiments described in this manuscript. Each provided informed written consent and was paid \$AUD 25 per hour for their participation. All had normal or corrected-to-normal vision. Experiments were approved by the University of Western Sydney's Human Research Ethics committee (H8862) and were conducted in accordance with the Code 
of Ethics of the World Medical Association (Declaration of Helsinki) for experiments involving humans.

\section{Apparatus, stimuli, and procedure}

This experiment involved an arrangement of four target/ distractor ensembles, identical in most respects to that used in Experiment 1 (target eccentricity $=8^{\circ}$ of visual angle) with the following exceptions. The global arrangement of the target/ distractor ensembles was rotated 45 degrees (centred on fixation), producing the stimulus configurations seen in Fig. $6 \mathrm{a}-\mathrm{b}$. Thus, two targets (each surrounded by its corresponding set of 10 distractors) were horizontally distributed $5.66^{\circ}$ degrees above fixation, and two were horizontally distributed $5.66^{\circ}$ below fixation (see horizontal ellipses in Fig. 5). Similarly, two sets of target/distractor sets were vertically distributed; one $5.66^{\circ}$ left of fixation, the other $5.66^{\circ}$ to the right (see vertical ellipses in Fig. 5). The SOAs used in Experiment 3 were $-94 \mathrm{~ms},-59 \mathrm{~ms}$, $-35 \mathrm{~ms},-12 \mathrm{~ms}, 12 \mathrm{~ms}, 35 \mathrm{~ms}, 59 \mathrm{~ms}$, and $94 \mathrm{~ms}$ for static distractor trials; $-294 \mathrm{~ms},-247 \mathrm{~ms},-94 \mathrm{~ms},-35 \mathrm{~ms}, 35 \mathrm{~ms}, 94$ $\mathrm{ms}, 247 \mathrm{~ms}$, and $294 \mathrm{~ms}$ for dynamic distractor trials.

Like Experiment 1, on each trial all four target-distractor ensembles were presented on the screen simultaneously. However, within an experimental block only vertically arranged or horizontally arranged target events were presented. Observers were informed prior to a given experiment block whether targets would be horizontally or vertically arranged. Although trials of a given target orientation were blocked, unlike Experiment 2, target events could occur either side of fixation. That is to say, for a vertical block of trials, whereas trial $n$ could involve luminance transitions in a pair of targets to the left of fixation, followed by trial $n+1$, in which the target pair could appear to the right. This target location varied randomly from trial to trial. Within an experimental block, distractor context (static vs dynamic distractors) was randomised from trial to trial.

Observers were instructed to register the temporal order of horizontally displaced target events (occurring above or below fixation) using the left and right arrow keys on a computer keyboard. Conversely, up and down arrow keys were used to register the temporal sequence of vertically displaced target events (occurring left or right of fixation).

Each experimental block contained a total of 256 trials within which the virtual trajectory of a given pair of targets was either horizontal (within upper and lower visual fields) or vertical (within left and right visual fields). Different levels of distractor context (static vs dynamic), target SOA and target location (above or below fixation for horizontal blocks, left or right of fixation for vertical blocks) were randomised across trials. Each observer performed four blocks of 256 trials (1,024 trials per observer). Block order was counterbalanced across observers.

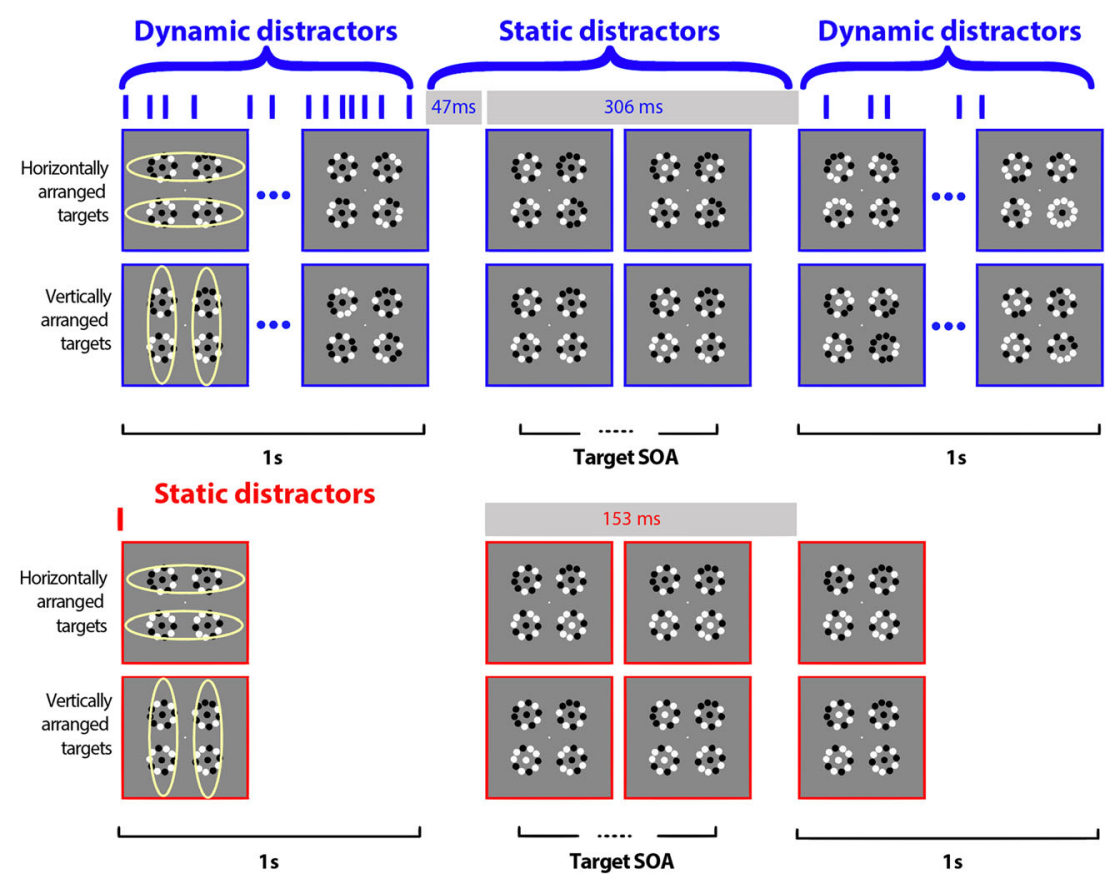

Fig. 5 Example trial sequence representing each of the two distractor conditions and target locations used in Experiment 3: dynamic context (blue); static context (red). The yellow ellipses in the left panelspresented here for illustrative purposes - signify whether the trial involved target events located above or below fixation (horizontal [left/right first] judgments) or left or right of fixation (vertical [top/bottom first] judgments). The orientation of the target judgment was blocked across trials. Observers were informed of the orientation of the target events prior to each block. Blue and red vertical bars above the stimulus configuration panels signify distractor events. (Colour figure online) 


\section{Results}

A chi-square analysis was conducted on each individual participant's data (averaged across SOA) to estimate the goodness of fit for each experimental condition. Respectively, the average $\chi^{2}$ and $p$ values ( 7 degrees of freedom) obtained in each condition were: Static left, $\chi^{2}=16.2, p=.014$; static right, $\chi^{2}=14.6, p=$ .024 ; static top, $\chi^{2}=14.8, p=.021$; static bottom, $\chi^{2}=14.2, p=$ .026 ; dynamic left, $\chi^{2}=11.2, p=.08$; dynamic right, $\chi^{2}=11.4, p$ $=.08$; dynamic top, $\chi^{2}=11.2, p=.09$; dynamic bottom, $\chi^{2}=$ 10.4, $p=.10$. Like Experiments 1 and 2, a proportion of these psychometric fits failed to reach critical significance. ing the proportion of 'correct' responses for negative versus the various conditions. Note that these accuracy effects (reported below) qualitatively match the JND analyses below.

Just noticeable difference (JND) The results of Experiment 3 are shown in Fig. 6. Two participants were omitted from the group JND analyses due to extremely poor psychometric curve fits (yielding estimates in excess of 5,000 ms). For the remaining participants, a repeated-measures ANOVA comparing the effects of visual field and distractor context on fitted Consequently, we ran a series of confirmatory analyses comparpositive SOAs (omitting the two smallest absolute SOAs) in

JNDs (see Fig. 6c) shows that on average, dynamic distractors caused significant threshold elevation relative to static distractor environments, $F(1,11)=13.388, p=.004, \eta^{2}=$ .549. A significant main effect of visual field location was also observed, $F(3,11)=5.745, p=.003, \eta^{2}=.343$, indicating that TOJ performance differed significantly among the four different visual fields. A significant interaction was found between distractor context and visual field location, $F(3,11)=5.931, p$ $=.002, \eta^{2}=.350$. To deconstruct this interaction, two separate one-way repeated-measures ANOVAs were conducted; one ANOVA comparing the effects of visual field for static distractor environments, and for the other ANOVA, dynamic distractor environments. Whereas no significant differences were observed across visual field locations in static distractor environments, $F(3,11)=0.556, p=.648, \eta^{2}=.048$, a significant effect of visual field was observed in the dynamic distractor case, $F(3,11)=5.848, p=.003, \eta^{2}=.347$. Two separate pairwise comparisons (one-tailed Bonferroniadjusted repeated-measures $t$ tests) of visual field location were conducted on dynamic distractor-related thresholds. These analyses indicated that JNDs were significantly higher in upper relative to lower visual fields, $t(11)=2.575, p=.026$. No differences in JND were observed across left and right visual fields, $t(11)=0.554, p=.590$. Confirmatory repeated-

(b)
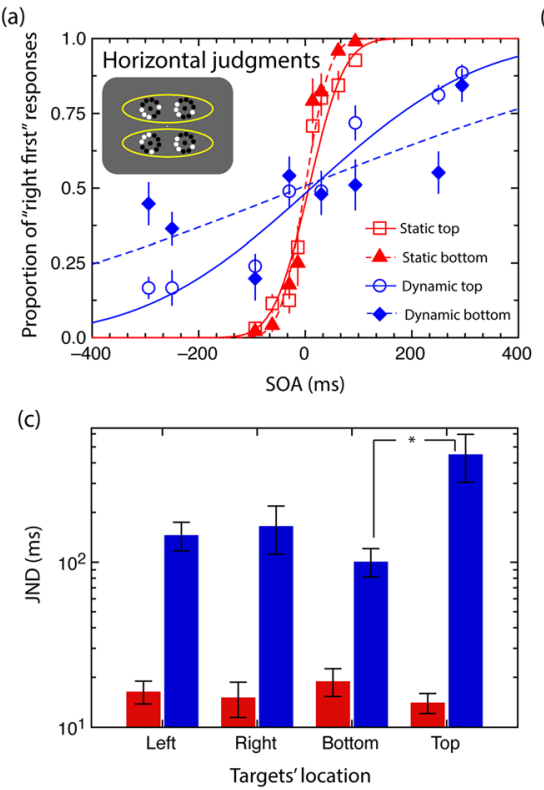

Fig. 6 Results of Experiment 3. a Proportion of 'right target first' responses as a function of target stimulus onset asynchrony (SOA) averaged across subjects for each of the two contextual conditions: static distractors (red circles); dynamic distractors (blue diamonds) for horizontal judgments. Curves are best-fitting cumulative Gaussians measured for each contextual condition shown here for illustrative purposes only. Statistical analyses were conducted on JNDs derived for each subject in each experimental condition for vertical judgments. b Proportion of 'bottom target first' responses as a function of target stimulus onset asynchrony (SOA) averaged across subjects for each of the two contextual conditions. Insets in Fig. 6a-b show an example stimulus frame, with the

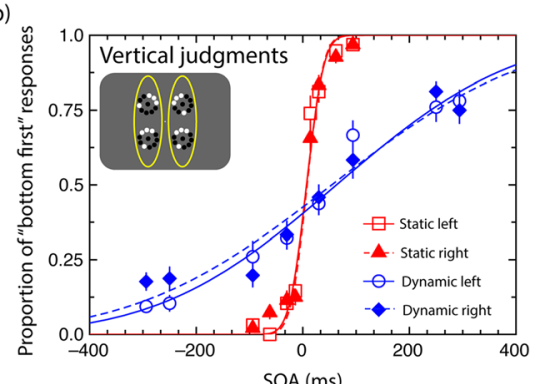

(d)

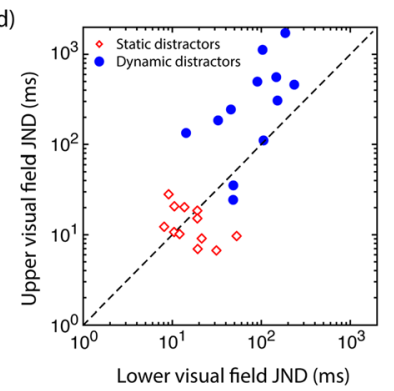

principal orientation of yellow ellipses corresponding to the orientation of the targets' virtual motion trajectory. c Average JNDs derived from individual subjects' fits in each contextual distractor and target trajectory orientation condition. Error bars represent between-subject mean standard error. d Comparison of individual observer JNDs measured above vs below fixation under dynamic (blue circles) and static (red diamonds) distractor conditions. Note that $\mathbf{d}$ shows the results for horizontal judgments only, and that the dynamic JNDs located above the diagonal indicate an upper-lower asymmetry for these observers. (Colour figure online) 
measures $t$ tests run on accuracy data in dynamic distractor conditions yielded qualitatively equivalent visual field effects: upper versus lower visual field, $t(11)=3.106, p=.010$; left versus right visual field, $t(11)=0.411, p=.689$.

For illustrative purposes, a comparison of JNDs derived for individual observers in upper versus lower visual fields is depicted in Fig. 6d.

An additional between-subjects comparison of thresholds measured in dynamic distractor-related thresholds (assigning subject as a random variable) failed to detect a significant difference between different target orientations (vertical $=$ left + right visual fields; horizontal $=$ upper vs lower visual fields), $F(1,11)=2.272, p=.176$.

Point of subjective simultaneity (PSS) A repeated-measures ANOVA on PSSs failed to find significant main effects of distractor context, $F(1,11)=0.042, p=.842, \eta^{2}=.004$, or visual field location, $F(3,11)=2.266, p=.150, \eta^{2}=.004$. No significant interaction between distractor context and meridian was observed, $F(3,11)=1.761, p=.224, \eta^{2}=.370$.

To determine, whether the PSS estimates for any of these conditions differed significantly from zero, we ran eight separate one-sample $t$ tests (all two-tailed). Of these analyses (see Table 3), four differed significantly from zero: static/left; static/right; dynamic/left; dynamic/right. It is worth noting that all of these significant shifts in PSS were for vertical judgments. That these shifts were all in a positive SOA direction implies a that observers were biased to perceive target events above fixation prior to target events below fixation.

\section{Discussion}

The results of Experiment 3 confirm that TOJs exhibit the predicted upper-lower anisotropy. That is to say, threshold elevation due to dynamic distractors was of significantly greater magnitude in the upper relative to the lower visual fields. By contrast, thresholds associated with static distractor environments were statistically indistinguishable at all locations tested. Again, this dissociation in the effects of visual

Table 3 Mean PSS estimates and results of one-sample t-tests showing statistical deviation of PSS estimates from zero milliseconds. Double and triple asterisks signify statistically significant $p$ values less than .025 and .005 , respectively. All $p$ values are corrected for multiple comparisons

\begin{tabular}{lllll}
\hline Distractor context & Visual field & Mean PSS (ms) & \multicolumn{1}{l}{$p$} \\
\hline Static & Left & 5.9 & 5.186 & $<.001 * * *$ \\
& Right & 7.5 & 6.272 & $<.001^{* * *}$ \\
& Lower & -8.1 & 0.886 & .394 \\
& Upper & -15.9 & -0.508 & .621 \\
Dynamic & Left & 59.6 & 4.367 & $.001 * * *$ \\
& Right & 42.1 & 3.133 & $.010^{* *}$ \\
& Lower & 7.9 & 0.443 & .666 \\
& Upper & -46.4 & -0.263 & .797 \\
\hline
\end{tabular}

field location and the distractor environment (static vs dynamic) implies that TOJs are likely to be mediated by independent subsystems.

Unexpectedly, we observed significant positive deviations from zero for PSS estimates in all conditions involving vertical TOJ judgments. That all exhibit a positive shift from zero implies an illusory but general bias to perceive the top disk as changing prior to the lower disk.

\section{General discussion}

This is the first study to demonstrate that TOJ performance is, under specific conditions, constrained by both of the classic visual field anisotropies (Abrams et al., 2012; Carrasco et al., 2004; Corbett \& Carrasco, 2011; He, Cavanagh, \& Intriligator, 1997; Intriligator \& Cavanagh, 2001; Talgar \& Carrasco, 2002). These are characterised as poorer temporal acuity: along the vertical relative to the horizontal meridianthe so-called 'lateral anisotropy' (Experiments 1 \& 2); and in upper relative to lower visual fields - an 'upper-lower anisotropy' (Experiment 3).

Critically, in all of our experiments, these visual field anisotropies were evident only in dynamic distractor environments. TOJs performed in static environments-which are dynamically equivalent to classic TOJ stimuli (see Cass \& Van der Burg, 2014, for similar performances in a target alone condition vs. static condition)-failed to produce a strong or consistent anisotropy. Why do dynamic distractor environments produce a qualitative pattern of performance consistent with the classic anisotropies found in numerous nontemporal tasks such as visual search, object individuation, orientation, and contrast discrimination (Abrams et al., 2012; Corbett \& Carrasco, 2011; He et al., 1996; Intriligator \& Cavanagh, 2001; Talgar \& Carrasco, 2002), whereas static distractor environments do not?

A simple explanation may be that performance under static distractor conditions may be too close to ceiling levels to produce statistically significant visual field anisotropies of the kind observed in dynamic distractor environments. The very low threshold estimates, ranging between $6 \mathrm{~ms}$ and 40 $\mathrm{ms}$, combined with the small between-subject standard deviations $(\sim 15 \mathrm{~ms})$ observed in static distractor environments suggests that this may be the case. This interpretation may be premature, however, when considering the anisotropic performance observed under static distractor conditions in Experiment 1, in which thresholds were lower along the vertical than along the horizontal meridian - the opposite anisotropy to what is observed in dynamic distractor environments. This dissociation between the effects of visual meridian and distractor type suggests the involvement of multiple motion discrimination processes. 
To borrow from Holcombe's dual processing scheme of temporal vision, we propose that standard TOJ tasks (and those measured here in static distractor environments) may depend upon the response of low-order direction-selective motion mechanisms, which afford relatively high temporal resolution. Indeed, TOJ thresholds measured here in static distractor environments yield JNDs of approximately $25 \mathrm{~ms}$; a value falling within the range of Holcombe's 'fast' visual system (Holcombe, 2009). By contrast, the thresholds observed in dynamic distractor environments (mean JNDs ranging from $83 \mathrm{~ms}$ to $441 \mathrm{~ms}$ ) are consistent with a qualitative shift to the 'slower' (lower temporal resolution) and attentionally demanding motion-tracking system. To account for this shift, we propose that dynamic distractors introduce a source of low-order motion noise at either sensory and/or decisional levels of processing, precluding — at least partiallyperceptual access to task-relevant low-order motion signals. Consequently, we assume that TOJ performance in our dynamic distractor conditions reflects a reliance on the higher order motion-tracking system, which is associated with being both temporally sluggish and attentionally demanding (Aghdaee \& Cavanagh, 2007; Holcombe, 2009).

That the TOJ performance anisotropies observed in Experiments 1-3 qualitatively match those reported in a range of nontemporal tasks suggests a common process, possibly involving attention. This interpretation contrasts with a previous report which found no correspondence between the effects of visual field for a task assumed to signify 'temporal attention' (long-range temporal phase discrimination; Aghdaee \& Cavanagh, 2007) and the well-established anisotropies associated with other attentionally demanding, but nontemporal tasks (He et al., 1996; Intriligator \& Cavanagh, 2001). Although our data appear consistent with the linking of high-order attentional tracking performance with other nontemporal attentionally demanding tasks, we advise caution in this interpretation. Firstly, not all attentionally demanding tasks exhibit the classical anisotropies. Covert spatial attention affects discriminability at isoeccentric visual field locations to a similar degree (Cameron et al., 2002; Carrasco et al., 2001; Roberts, Cymerman, Smith, Kiorpes, \& Carrasco, 2016). Moreover, there is currently no definitive evidence that the TOJ performance observed in dynamic distractor conditions - and concomitant threshold elevationnecessarily implies reliance on high-order attentional tracking processes. Future studies might investigate this using an attentionally demanding dual-task equivalent noise paradigm, comparing the effects of attention on TOJ performance across various levels of distractor noise.

Despite the qualitative agreement between the lateral and upper lower anisotropies observed here in dynamic distractor conditions and the numerous nontemporal tasks reported elsewhere, the question remains as to why static environments yielded superior performance along the vertical meridian in
Experiment 1. This finding appears at odds with the predicted lateral anisotropy by which the horizontal meridian is expected to afford lower thresholds. Notably, no such anisotropy was observed under static distractor conditions in Experiment 2, suggesting a possible role for uncertainty. Future studies may test this by systematically varying the likelihood that targets will appear along either the horizontal or the vertical meridian or, indeed in other regions of the visual field.

An unforeseen implication of these results pertains to the absence of an overall orientation effect for JNDs in Experiment 3. As mentioned above, when viewing natural scenes, humans elicit a bias favouring horizontal scanning paths. That no such bias was observed for JNDs in Experiment 3, yet was observed in Experiments 1 and 2, suggests that trajectory orientation is unlikely to be the sole factor driving the observed meridian effects.

Despite the absence of an orientation effect for JNDs, Experiment 3 did yield positive shifts in PSS specific to vertical judgments. This implies that observers were systematically biased towards perceiving change in the upper disk within a vertically arranged pair prior to change in the lower disk. This appears to contradict the law of prior entry, which states that regions of the visual field which afford higher levels of attention will speed up processing (Spence \& Parise, 2010). Given the numerous studies indicating that attentional processes are superior in the lower relative to upper visual fields (e.g. He et al., 1997; Intriligator \& Cavanagh, 2001), one might expect to observe the opposite PSS anisotropy to that observed here. In terms of motion, our observed positive PSS shifts correspond to a bias favouring downward motion trajectories. Future research might investigate whether the PSS anisotropy observed in Experiment 3 reflects gravitational constraints (i.e. tendency to perceive downward motion) or a purely temporal (upper visual field) processing bias. We also recommend that future studies measure eye movements to determine whether these upper visual field biases may be linked to systematic changes in eye position.

One factor which distinguishes Experiment 3 from Experiments 1 and 2 is that the targets were located in the noncardinal visual quadrants (i.e. diagonally with respect to fixation) rather than along the cardinal (meridian) axes. Previous studies have shown that performance in these noncardinal locations is generally poorer than along the meridians (Corbett \& Carrasco, 2011). Aside from the higher thresholds specific to the upper visual field, we find that, in general, performance associated with the noncardinal locations (Experiment 3) was indistinguishable from the meridian locations (Experiment 1). To our knowledge, the performance fields literature has not reported the results of tasks involving perceptual comparison of objects or events located between noncardinal axes. Future research is necessary to establish whether the intercardinal TOJ performance we observe (in Experiment 3) generalises to other tasks. 


\section{Conclusion}

This is the first study to demonstrate that visual TOJ acuity is constrained by a set of visual field anisotropies qualitatively identical to those evidenced in a range of nontemporal tasks, such as visual search, contrast and orientation discrimination, and crowding (Corbett \& Carrasco, 2011; He et al., 1996; Yeshurun \& Carrasco, 1999). Critically, these lateral and upper-lower anisotropies were only observed when employing dynamic distractors, with performance in static distractor environments approximately isotropic across the visual field; and in the case of Experiment 1, the opposite anisotropy. This general pattern of results is consistent with Holcombe's dual temporal processing scheme, whereby 'high-level' motion tracking tasks are constrained by processes affording poorer temporal resolution than 'low-level' temporal tasks, including flicker and apparent motion perception. The results of our experiments indicate that it is these high-level motion tasks which are likely to be constrained by the classic lateral and upper-lower visual field anisotropies.

Publisher's Note Springer Nature remains neutral with regard to jurisdictional claims in published maps and institutional affiliations.

\section{References}

Abrams, J., Nizam, A., \& Carrasco, M. (2012). Isoeccentric locations are not equivalent: The extent of the vertical meridian asymmetry. Vision Research, 52(1), 70-78.https://doi.org/10.1016/j.visres. 2011.10.016

Aghdaee, S. M., \& Cavanagh, P. (2007). Temporal limits of long-range phase discrimination across the visual field. Vision Research, 47(16), 2156-2163. https://doi.org/10.1016/j.visres.2007.04.016

Cameron, E. L., Tai, J. C., \& Carrasco, M. (2002). Covert attention affects the psychometric function of contrast sensitivity. Vision Research, 42(8), 949-967.

Carrasco, M., Evert, D. L., Chang, I., \& Katz, S. M. (1995). The eccentricity effect: Target eccentricity affects performance on conjunction searches. Perception \& Psychophysics, 57(8), 1241-1261.

Carrasco, M., \& Frieder, K. S. (1997). Cortical magnification neutralizes the eccentricity effect in visual search. Vision Research, 37(1), 63-82.

Carrasco, M., Giordano, A. M., \& McElree, B. (2004). Temporal performance fields: Visual and attentional factors. Vision Research, 44(12), 1351-1365. https://doi.org/10.1016/j.visres.2003.11.026

Carrasco, M., Talgar, C. P., \& Cameron, E. L. (2001). Characterizing visual performance fields: Effects of transient covert attention, spatial frequency, eccentricity, task and set size. Spatial Vision, 15(1), $61-75$.

Cass, J., \& Van der Burg, E. (2014). Remote temporal camouflage: Contextual flicker disrupts perceived visual temporal order. Vision Research, 103, 92-100. https://doi.org/10.1016/j.visres.2014.08. 008

Chaikin, J. D., Corbin, H. H., \& Volkmann, J. (1962). Mapping a field of short-time visual search. Science, 138(3547), 1327-1328.

Corbett, J. E., \& Carrasco, M. (2011). Visual performance fields: Frames of reference. PLOS ONE, 6(9), e24470. https://doi.org/10.1371/ journal.pone. 0024470
Curcio, C. A., \& Allen, K. A. (1990). Topography of ganglion cells in human retina. The Journal of Comparative Neurology, 300(1), 5-25. https://doi.org/10.1002/cne.903000103

de Lange, H. (1952). Experiments on flicker and some calculations on an electrical analogue of the foveal systems. Physica, 18, 935-950.

de Lange, H. (1958). Research into the dynamic nature of the human fovea-cortex systems with intermittent and modulated light. I. Attenuation characteristics with white and colored light. Journal of the Optical Society of America. A, 48(11), 777-784.

Forte, J., Hogben, J. H., \& Ross, J. (1999). Spatial limitations of temporal segmentation. Vision Research, 39(24), 4052-4061.

Foulsham, T., Kingstone, A., \& Underwood, G. (2008). Turning the world around: Patterns in saccade direction vary with picture orientation. Vision Research, 48(17), 1777-1790. https://doi.org/10.1016/ j.visres.2008.05.018

Golla, H., Ignashchenkova, A., Haarmeier, T., \& Thier, P. (2004). Improvement of visual acuity by spatial cueing: A comparative study in human and non-human primates. Vision Research, 44(13), 1589-1600. https://doi.org/10.1016/j.visres.2004.01.009

He, S., Cavanagh, P., \& Intriligator, J. (1996). Attentional resolution and the locus of visual awareness. Nature, 383(6598), 334-337. https:// doi.org/10.1038/383334a 0

He, S., Cavanagh, P., \& Intriligator, J. (1997). Attentional resolution. Trends in Cognitive Science, 1(3), 115-121. https://doi.org/10. 1016/S1364-6613(97)89058-4

Holcombe, A. O. (2009). Seeing slow and seeing fast: Two limits on perception. Trends in Cognitive Science, 13(5), 216-221. https:// doi.org/10.1016/j.tics.2009.02.005

Intriligator, J., \& Cavanagh, P. (2001). The spatial resolution of visual attention. Cognitive Psychology, 43(3), 171-216. https://doi.org/10. 1006/cogp.2001.0755

Kristjansson, A., \& Sigurdardottir, H. M. (2008). On the benefits of transient attention across the visual field. Perception, 37(5), 747-764. https://doi.org/10.1068/p5922

Levi, D. M., \& Waugh, S. J. (1994). Spatial scale shifts in peripheral vernier acuity. Vision Research, 34(17), 2215-2238.

Lim, A., \& Sinnett, S. (2012). Reexamining visual orientation anisotropies: A bias towards simple horizontal stimuli on temporal order judgments. Austin, TX: Cognitive Science Society.

Maruya, K., Holcombe, A. O., \& Nishida, S. (2013). Rapid encoding of relationships between spatially remote motion signals. Journal of Vision, 13(2), 4. https://doi.org/10.1167/13.2.4

Olivers, C. N., Awh, E., \& Van der Burg, E. (2016). The capacity to detect synchronous audiovisual events is severely limited: Evidence from mixture modeling. Journal of Experimental Psychology: Human Perception and Performance, 42(12), 2115-2124. https://doi.org/ $10.1037 / \mathrm{xhp} 0000268$

Perry, V. H., \& Cowey, A. (1985). The ganglion cell and cone distributions in the monkey's retina: Implications for central magnification factors. Vision Research, 25(12), 1795-1810.

Raninen, A., \& Rovamo, J. (1997). Flicker sensitivity as a function of eccentricity. Perception, 26(1 Suppl), 327-327.

Rijsdijk, J. P., Kroon, J. N., \& van der Wildt, G. J. (1980). Contrast sensitivity as a function of position on the retina. Vision Research, 20(3), 235-241.

Roberts, M., Cymerman, R., Smith, R. T., Kiorpes, L., \& Carrasco, M. (2016). Covert spatial attention is functionally intact in amblyopic human adults. Journal of Vision, 16(15), 30. https://doi.org/10.1167/ 16.15.30

Rodríguez-Sánchez, A., Fallah, M., \& Leonardis, A. (2016). Hierarchical object representations in the visual cortex and computer vision. Frontiers in Computation Neuroscience. https://doi.org/10.3389/ fncom.2015.00142.

Rogers-Ramachandran, D. C., \& Ramachandran, V. S. (1998). Psychophysical evidence for boundary and surface systems in human vision. Vision Research, 38(1), 71-77. 
Rovamo, J., \& Raninen, A. (1984). Critical flicker frequency and Mscaling of stimulus size and retinal illuminance. Vision Research, 24(10), 1127-1131.

Seiple, W., Holopigian, K., Szlyk, J. P., \& Wu, C. (2004). Multidimensional visual field maps: Relationships among local psychophysical and local electrophysiological measures. Journal of Rehabilitation Research and Development, 41(3A), 359-372.

Spence, C., \& Parise, C. (2010). Prior-entry: A review. Conscious Cognition, 19(1), 364-379. https://doi.org/10.1016/j.concog.2009.12.001

Talbot, D., Van der Burg, E., \& Cass, J. (2017). Stereoscopic segmentation cues improve visual timing performance in spatiotemporally cluttered environments. i-perception, 8(2), 2041669517699222. https://doi.org/10.1177/2041669517699222

Talgar, C. P., \& Carrasco, M. (2002). Vertical meridian asymmetry in spatial resolution: Visual and attentional factors. Psychonomic Bulletin \& Review, 9(4), 714-722.
Tyler, C. W. (1987). Analysis of visual modulation sensitivity. III. Meridional variations in peripheral flicker sensitivity. Journal of the Optical Society of America A, 4(8), 1612-1619.

Van der Burg, E., Awh, E., \& Olivers, C. N. (2013). The capacity of audiovisual integration is limited to one item. Psychological Science, 24(3), 345-351. https://doi.org/10.1177/0956797612452865

Virsu, V., \& Rovamo, J. (1979). Visual resolution, contrast sensitivity, and the cortical magnification factor. Experimental Brain Research, 37(3), 475-494.

Westheimer, G. (1983). Temporal order detection for foveal and peripheral visual stimuli. Vision Research, 23(8), 759-763.

Yasuma, T., Miyakawa, N., \& Yamazaki, J. (1986). Clinical application of time-dependent perimetry: 1. Results in normal subjects. Japanese Journal of Ophthalmology, 30(3), 330-337.

Yeshurun, Y., \& Carrasco, M. (1999). Spatial attention improves performance in spatial resolution tasks. Vision Research, 39(2), 293-306. 